\title{
On the Design of Miniature Haptic Devices for Upper Extremity Prosthetics
}

\author{
Keehoon Kim, J. Edward Colgate, Julio J. Santos-Munné, Alex Makhlin, Michael A. Peshkin
}

\begin{abstract}
We have developed three different versions of a multi-function haptic device that can display touch, pressure, vibration, shear force, and temperature to the skin of an upper extremity amputee, especially one who has undergone targeted nerve reinnervation (TR) surgery. In TR patients, sensation from the reinnervated skin is projected to the missing hand. This paper addresses the design of the mechanical display, the portion responsible for contact, pressure, vibration, and shear force. A variety of different overall design approaches satisfying the design specifications and the performance requirements are considered. The designs of the fully prototyped haptic devices are compared through open-loop frequency response, closed loop force response, and tapping response in constrained motion. We emphasize the tradeoffs between key design factors including force capability, workspace, size, bandwidth, weight, and mechanism complexity.
\end{abstract}

Index Terms - multi function tactor, miniature haptic device, mechanical haptic display, upper extremity prosthesis, haptic feedback

\section{INTRODUCTION}

The design of upper extremity prostheses often gives precedence to cosmesis, grasping, and manipulation. Also important, however, is haptic perception. Haptics subserves a wide variety of manual activities including stable grasping, activating buttons, knobs and other interface devices, detecting shape, compliance and texture, and so on. The problem of restoring haptic perception to an amputee, however, is a difficult one Human skin, especially the glabrous skin of the fingertips, is richly innervated by a variety of specialized mechanoreceptors and free nerve endings. The musculoskeletal system is also rich in sensors. All of this information is lost when a limb is amputated. While a prosthetic device can be outfitted in sensors (though certainly not the number of sensors found in the intact limb), it is by no means clear how best to convey sensor data to the nervous system.

It seems natural that, in order to achieve intuitive haptic feedback, it is beneficial to satisfy two conditions: $i$ ) somatotopic matching and ii) modality matching. For example, when

This research was performed as part of the Johns Hopkins University Applied Physics Laboratory (JHUAPL) Revolutionizing Prosthetics 2009 funded by the Defense Advanced Research Projects Agency (DARPA) Contract \# N66001-06-C-8005. This material is based upon work supported by, or in part by, the U. S. Army Research Laboratory and the U. S. Army Research Office under contract/grant number \#W911NF-06-C-0001. Parts of this paper were presented at the 2007 Frontiers in the Convergence of Bioscience and Information Technologies (FBIT 2007), Special Session on Haptics, Jeju, Korea, October 11-13.

Keehoon Kim, J. Edward Colgate, and Michael A Peshkin are with the Department of Mechanical Engineering, Northwestern University, Evanston, IL 60091, USA khkapostech.ac.kr, colgatednorthwestern.edu, peshkin@northwestern.edu

Julio J. Santos-Munné and Alex Makhlin are with Kinea Design, LLC, Evanston, IL 60201, USA jsantos@kineadesign.com, amakhlinekineadesign. com we press an object with the prosthetic thumb, if we feel the pressure at the thumb, then we can say that modality (pressure) and somatotopic (thumb) matching conditions are satisfied. Our principal research goal is to provide amputees with a somatotopically matched haptic perception system for the modalities of touch, pressure, vibration, shear, and temperature (i.e., we focus on tactile sensation rather than kinesthetic sensation). We are also concerned about practical issues such as energy consumption, ease of donning and doffing, comfort, and cosmesis.

The problem of providing haptic feedback to amputees has been approached in a number of different ways. A straight forward approach is "sensory substitution" in which neither somatotopic nor modality matching is attempted [1]. For instance, vibration and electrocutaneous displays mounted on various parts of the body including the forearm, abdomen, and back have been used to represent tactile sensation, proprioception, or the grip pressure of a prosthetic hand [2]-[4]. Although this method may be used to realize miniature and reliable haptic displays, it is by no means evident that the results are natural, intuitive, or desirable to amputees.

Modality-matched displays of grip pressure have also been developed [5]-[8]. For instance, Meek et al. explored grip pressure feedback with a myoelectriclly controlled prosthetic arm. They mounted a servo-controlled "pusher" to the socket. The pusher pressed into the skin an amount proportional to the force in the terminal device, a method that the authors termed "Extended Physiologic Taction" (EPT). It was reported that the ability to grip a brittle object without breaking it was improved with EPT, but task completion time was not [5].

Recently, Kuiken et al. developed Targeted Reinnervation (TR) surgery that reroutes severed nerves from the amputated limb to residual muscles and skin [9]-[12]. In this surgery, both the efferent and the afferent nerves are rerouted to the residual muscles and the skin, respectively. Thus, when the TR patient thinks about moving his or her missing limb, efferent nerve signals contract the residual muscles. EMG signals from these muscles can be used as command signals for a prosthetic arm. In effect, muscle is being used both to keep the residual nerves healthy and to serve as an amplifier of neural signals. Reinnervation also creates sensory regions on the patient's skin (for instance, the chest or upper arm) that are referred to the surface of the missing limb. As a result, TR 


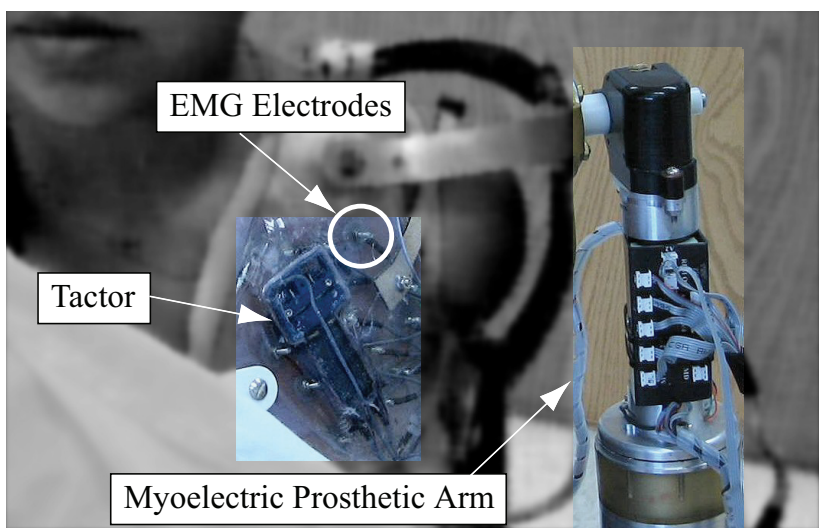

Fig. 1. Tactor, EMG electrodes, and a myoelectric prosthetic arm on a shoulder disarticulation TR patient. The system operates as follows: 1) Efferent nerves signal the residual muscles to contract; 2) EMG activity associated with these muscles is measured and used as command signals for the myoelectric prosthetic arm; 3) Sensors placed on the prosthetic hand detect tactile stimuli; 4) These stimuli are "played back" to reinnvervated skin, creating intuitive haptic feedback.

provides a practical means of somatotopic matching. ${ }^{1}$ In other words, it should be possible to create intuitive haptic feedback system by placing sensors on the prosthetic hand ( [13], [14]) and using these to drive corresponding (somatotopically matched) haptic displays on the TR skin as shown in Fig 1. Modality matching is also possible with TR patients. At least some patients perceive a variety of stimuli in a normal manner: pressure applied to the reinnervated skin is perceived as pressure on the hand; vibration maps to vibration; hot/cold map to hot/cold; and sharp objects can be distinguished from dull [15].

In this paper, we report on our efforts to design multifunction tactors that can deliver a variety of stimuli to the skin of a TR patient (or a non-TR patient, although in this case we cannot achieve somatotopic matching). There are many trade-offs to consider when designing tactors. Toward this end, we have developed three different designs (Fig. 2) (each of these designs is described in more detail in Section IV-A), each emphasizing somewhat different needs. We report on the performance of each design, emphasizing the trade-offs. Note, however, that we do not report on any experiments performed with amputees. Such experiments rely on advanced prosthetic hands and controllers, as well as extensive system integration, and will be the subject of future papers. In the next section, we begin with a discussion of the design specifications and the performance requirements for multi-function tactors.

\footnotetext{
${ }^{1}$ To be more precise, we refer to this as "weak somatotopic matching." The word "somatotopy" generally refers to an organized and connected map such that adjacent regions on the skin are represented on adjacent regions of the somatotopic cortex. We call this "strong somatotopic matching." But it is not the case for TR patients. While somatotopy still applies to the sensations that are referred to the missing hand, it does not apply to the reinnervated skin because the mapping is no longer organized and connected. Adjacent regions on the reinnervated skin are not necessarily represented on adjacent regions of the cortex.
}

TABLE I

Mechanical Performance Requirements of a TACtor

\begin{tabular}{l|c|l}
\hline \multirow{2}{*}{ Pressure \& Shear } & maximum force & $8.8 \mathrm{~N}$ \\
\cline { 2 - 3 } & resolution & $0.1 \mathrm{~N}$ \\
\hline \multirow{2}{*}{ Vibration } & maximum velocity & $0.025 \mathrm{~m} / \mathrm{sec}$ \\
\cline { 2 - 3 } & maximum acceleration & $16.0 \mathrm{~m} / \mathrm{sec}^{2}$ \\
\hline \multirow{2}{*}{ Tapping } & maximum velocity & $0.4 \mathrm{~m} / \mathrm{sec}^{2}$ \\
\cline { 2 - 3 } & maximum acceleration & $40.0 \mathrm{~m} / \mathrm{sec}^{2}$ \\
\hline Workspace & $10.0 \mathrm{~mm}$ for both pressure and shear \\
\hline Motion & Straight line path into skin preferable \\
\hline Tactor head size & $8.0 \mathrm{~mm}$ diameter \\
\hline
\end{tabular}

\section{Design Specifications And Performance REQUIREMENTS}

Because haptics comprises such an extraordinarily rich set of sensory pathways, it was important for our team to prioritize those which we wished to restore. Discussion led to the following prioritization.

First and foremost, we seek to restore the sense of contact with an object. This sense provides goal attainment information which is critical in multi-finger grasping. Second, we seek to restore the sense of pressure, which is critical in adjusting grip strength. Third is vibration, which is essential in discriminating among different textures. Fourth is temperature, which is useful both for material identification ( [16], [17]) and affective reasons: amputees often indicate the desire to feel the warmth of a loved one's touch. Fifth, is shear force, which is important in detecting slippage and in materials identification. Shear is especially important in discriminating slippery from sticky materials. ${ }^{2}$ Finally we seek to restore fine shape discrimination, such as the ability to feel an edge, separate sharp from dull, or read a set of Braille dots. In the work presented here, we explicitly address the first three goals as well as the fifth. Temperature is discussed elsewhere [18]. Fine shape discrimination is reserved for future work, as it will most likely require a different technical approach. We've determined from preliminary tests to be mentioned in section VI that this prioritization is reasonably well matched to the TR patients' desires.

At the outset of this project, we did not have quantitative specifications for any of the goals above since TR surgery had only recently become feasible. To establish the specifications, we began by building an experience prototype. The experience prototype was in no way intended to meet packaging, weight or power consumption requirements, but it was designed to apply forces and motions in response to sensor readings such that the motions, forces and power associated with various sensations could be measured. Fig. 3 shows the prototype which has a voice coil motor, two 3-axis force sensors (DX-46x, Bokam) and an 3-axis accelerometer (LIS3L06AL, STMicroelectronics).

The experience prototype was used to measure the force and motion associated with steady pressure, with tapping (the type of contact most demanding of tactors), and with vibrations

\footnotetext{
${ }^{2}$ Slippage detection is extremely important for prosthetic hands to ensure stable grasps, but anti-slip feedback loops can be closed locally without shear force feedback to the amputee.
} 


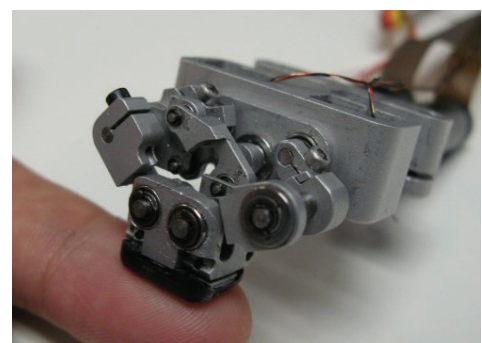

(a)

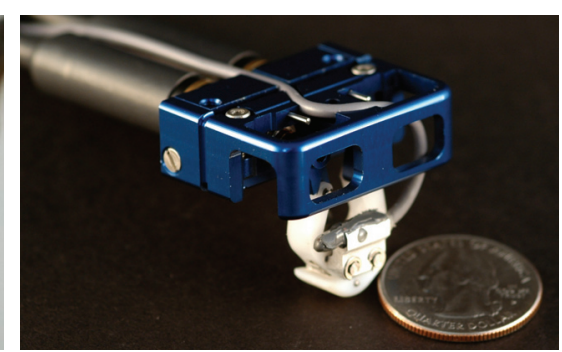

(b)

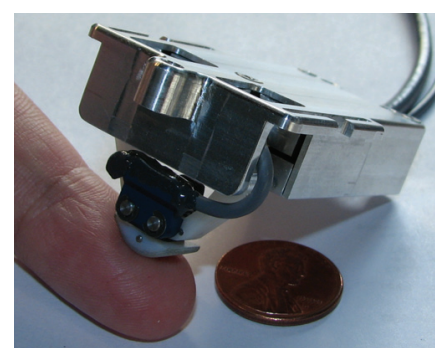

(c)

Fig. 2. Multi-function tactors which can display the sense of contact, pressure, vibration, shear force, and temperature: (a) 6-bar mechanism with skewed parallelogram constraint and $10 \mathrm{~mm}$ brushed motor (P10), (b) 6-bar mechanism with gear constraint and 10 mm brushed motor (G10), (c) 6-bar mechanism with gear constraint and $6 \mathrm{~mm}$ brushless motor (G6). (Please see Fig. 8 for detailed explanation of the parts.)

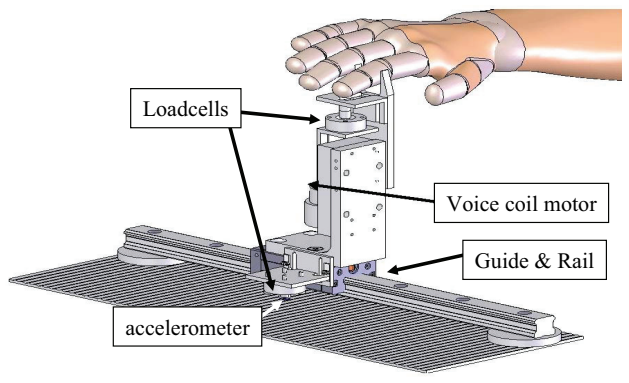

Fig. 3. A prototype to measure the force and motion associated with steady pressure, with tapping, and with vibrations caused by rubbing across various surfaces including different roughness ribbon cables and sandpapers.

caused by rubbing across various surfaces including different roughness ribbon cables and sandpaper. Vibration required up to $0.025 \mathrm{~m} / \mathrm{sec}$ and $16 \mathrm{~m} / \mathrm{sec}^{2}$. We found, however, that tapping demanded more of the tactor than vibration. Tapping required speeds of $0.4 \mathrm{~m} / \mathrm{sec}$, acceleration of $40 \mathrm{~m} / \mathrm{s}^{2}$, and forces of nearly $2 \mathrm{~N}$.

Though it did not prove practical to take this data with TR patients from a scheduling standpoint, these values make sense compared to the previous vibration threshold studies that will be explained with the experimental results in Section V. We were, however, able to take advantage of preliminary data from Kuiken et al. establishing peak force and displacement requirements for a TR patient [15], [19]. Pressure data indicated that one TR patient began to feel discomfort at $5.8 \mathrm{~N}$ normal force and pain at $8.8 \mathrm{~N}$ corresponding to skin penetration of about $10 \mathrm{~mm}$ with a $8.0 \mathrm{~mm}$ diameter tactor head. This same subject had a normal force JND (Just Noticeable Difference) of $0.15-0.2 \mathrm{~N}$. To ensure adequate range, we set the tactor maximum force specification at $9.0 \mathrm{~N}$, and the stroke at 10 $\mathrm{mm}$. In order to ensure safety, software and hardware limits are used in practice. Table I summarizes the key performance specifications.

In addition, we discovered that it was preferable for the tactor head to move straight down into the skin rather than follow a rotary path, and we found by trial and error that a tactor head about $8.0 \mathrm{~mm}$ in diameter provided a good tradeoff between power consumption (better for smaller heads) and comfort (better for larger heads).

A number of other constraints were placed on the design. The tactor was required to be light enough to be a wearable device. A compact profile was sought for aesthetic purposes. To maximize bandwidth, every effort was made to minimize friction and backlash. Ease of donning and doffing were required, as well as resistance to spills and sweat.

\section{Literature Review: Miniature Haptic Displays}

There are many possible approaches to meeting the design specifications and performance requirements discussed above. In this section, a review of the literature shows a number of clever approaches to conveying haptic sensations with small devices.

For instance, electro-tactile devices offer a potentially very compact and light weight approach [20]-[23]. However, it has recently been argued that "this stimulation method has the fundamental limitations such as physical incapacity to selectively stimulate Pacinian corpuscles in the deeper tissue without the activation of the shallower receptors," [24], [25]. Thus, additional haptic displays such as a high frequency vibrator are required to stimulate Pacinian corpuscles in the deeper tissue. While interesting, this approach sets up a competition between the vibrator and electrodes for limited TR skin space. Moreover, the electrical stimulus can also interfere with the EMG signal from the TR muscle contraction, which is the principal signal being used to control the prosthesis movement. When sweat is present, electro-tactile devices are known to produce uncomfortable prickly or tingling sensations.

Piezoelectric bimorph arrays have been used to display fine texture through lateral skin deformation [26]-[33]. While a candidate for future extension to fine shape display, the piezoelectric bimorph has the major disadvantages of limited 
force and motion range, typically too faint to meet our pressure and shear specifications.

Another alternative is to combine a small tactile device with a kinesthetic display. A number of investigators have recently explored the combination of tactile and kinesthetic displays [22], [34]-[41]. The literature indicates that this type of display significantly enhances sensations such as rolling, sliding, and curvature [34], [37], [40]. In spite of these advantages, most kinesthetic force feedback devices are externally grounded while we require a device that can be body-mounted yet still display significant normal and shear motions and forces.

We concluded that, while the literature offers a number of valuable insights, it does not provide clear direction for the design of a multi-function tactor that would meet our needs. Thus, we have developed new body mountable miniature haptic devices satisfying the specifications and performance requirements discussed above and able to display contact, pressure, shear, and vibration simultaneously. Although we developed three different devices, each of them is essentially a small and light two degree-of-freedom (DOF) robot: one axis for the direction normal to the skin and another for the directions tangential to the skin.

\section{Design of Miniature Haptic Device}

A variety of different overall design approaches incorporating 2-DOF and satisfying the design specifications and the performance requirements were considered. This section addresses the mechanisms, actuators, and sensors of three tactors that were fully prototyped.

\section{A. Mechanism Design}

Before developing our tactor mechanisms, we considered an approach using linear actuators such as voice coils due to its attractive high bandwidth and the simplicity of converting the linear motion of the actuators to the 2-DOF motion of the end effector. However, all electromagnetic linear actuators including voice coils have intrinsically low force capabilities. Moreover, it is not realistic to use a reduction with a voice coil due to limited stroke. Thus, we decided to design a mechanism coverting rotary motion to 2-DOF linear motion using DC brushless, DC brushed, or ultrasonic motors for high efficiency as well as the possibility of miniaturization.

Though a 5-bar mechanism was considered due to its simplicity, it was eliminated since there was no place to put the tactor head. In addition, it was tricky to pick link lengths providing adequate separation of the two actuated joints as well as sufficient workspace with good manipulability.

An alternative is a 6 -bar mechanism with a 1 -DOF constraint. We've considered two different 6-bar mechanisms: one using a gear constraint, and one using a skewed parallelogram constraint. The gear constrained mechanism is simple and its kinematic properties are similar to the well-known 5-bar mechanism. A key advantage of the 6-bar is that the tactor head stays roughly parallel to the skin surface, whereas all of the links on a 5-bar rotate through large angles. The 6-bar is simple enough, however, that it should prove possible to make very small tactor heads if needed. A negative point is that the gear constraint can be expected to introduce undesirable friction.

The skewed parallelogram mechanism has the advantages of keeping the tactor head almost perfectly parallel to skin at any pose and also reducing friction by eliminating the gear constraint. However, the complexity of this design with many joints and links introduces other difficulties such as link collisions, backlash, and over-constraint problems. Since these two mechanisms have quite different mechanical characteristics, we believe that a careful comparison will help us understand the design tradeoffs, improving future designs.

Kinematic properties of the mechanisms (e.g., workspace and manipulability) are determined by the lengths of the links. In order to find the link lengths, a custom MATLAB simulator was written to compute and display the relevant properties. As a first step, we solved for the relative (i.e., nondimensional) link lengths that would minimize the condition number of the Jacobian (which we used as a manipulability index) across the workspace. Following this, the absolute value of the link lengths was determined to ensure a large enough workspace and to ensure that the distance $\left(l_{5}\right)$ between active joints $\left(q_{1}\right.$ and $q_{5}$ in Fig. 4) was greater than the motor diameter. We designed two gear constraint mechanisms which have different dimensions for $l_{5}$ since two different diameter actuators were used. Thus, we've designed three mechanisms: two 6 bar mechanisms with gear constraint and one 6 bar mechanism with a skewed parallelogram constraint. Those mechanisms are named G10 (gear constraint; $10 \mathrm{~mm}$ motor diameter), G6 (gear constraint; $6 \mathrm{~mm}$ motor diameter), and P10 (skewed parallelogram constraint; $10 \mathrm{~mm}$ motor diameter). Actuator selection will be discussed in Section IV-B. The manipulability plots for the three mechanisms are shown in Fig. 5. Except at the workspace boundaries, each mechanism has a condition number less than 5.0, which leads to reasonably isotropic motion and force.

Since the P10 mechanism contains complicated closed kinematic loops, it is necessary to consider over-constraint problems caused by joint axes that are misaligned during assembly or manufacture. We used carefully selected bearing configurations to avoid the over-constraint problem. For instance, in Fig. 4(b), consider the closed loop of $J_{1}-J_{2}-J_{3}$ $J_{11}-J_{4}-J_{5}$. Normally, one would use two bearings (solid line circle) at each of these joints to ensure pure revolute behavior and no off-axis wobble. Doing so in a closed loop, however, virtually guarantees binding due to over-constraint. Instead, we used only a single bearing (dotted line circle) at joints $J_{11}$ and $J_{4}$. This allows enough off-axis wobble to resolve the over-constraint while still preventing any wobble of the tactor head itself (the link connecting $J_{3}$ and $J_{11}$ ). Similarly, for the closed loops of parallelogram $J_{1}-J_{7^{-}} J_{9^{-}} J_{6}$ and $J_{7^{-}} J_{8^{-}}$ $J_{10^{-}} J_{3}$, single bearings are used for the joints of the triangle part $\left(J_{7}-J_{8}-J_{9}\right)$ to resolve over-constraint.

In order to fabricate the mechanisms, we used miniature bearings ( $3 \mathrm{~mm}(\mathrm{OD}) / 1 \mathrm{~mm}(\mathrm{ID})$, A7Y5MP0301, SDP-SI, and $5 \mathrm{~mm}(\mathrm{OD}) / 2 \mathrm{~mm}(\mathrm{ID}), \mathrm{SB} 682 \mathrm{ZZ}$, MISUMI) and precisionmachined linkages (Hoffman Machine). In addition, components that come into contact with the skin are Teflon-coated to minimize possible chafing or irritation. Link collisions and 


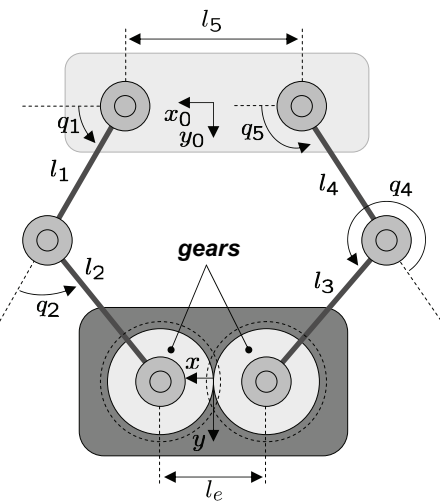

(a)

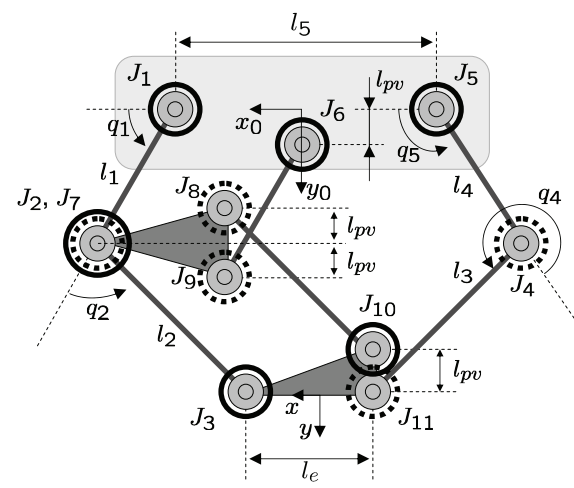

(b)

Fig. 4. Kinematic diagram. (a) 6 bar mechanism with gear constraint. $l_{1}=l_{4}=6.5 \mathrm{~mm}, l_{2}=l_{3}=11.5 \mathrm{~mm}$, and $l_{e}=4.75 \mathrm{~mm}$ for both G6 and G10. $l_{5}=11.0 \mathrm{~mm}$ for G10 and $l_{5}=9.5 \mathrm{~mm}$ for G6, (b) 6 bar mechanism with skewed parallelogram constraint and bearing configuration: double bearing joints (solid circle) and single bearing joints (dotted line circle). $l_{5}=12.0 \mathrm{~mm}, l_{1}=l_{4}=6.0 \mathrm{~mm}, l_{2}=l_{3}=9.6 \mathrm{~mm}$, and $l_{e}=6.0 \mathrm{~mm}$ for P10.

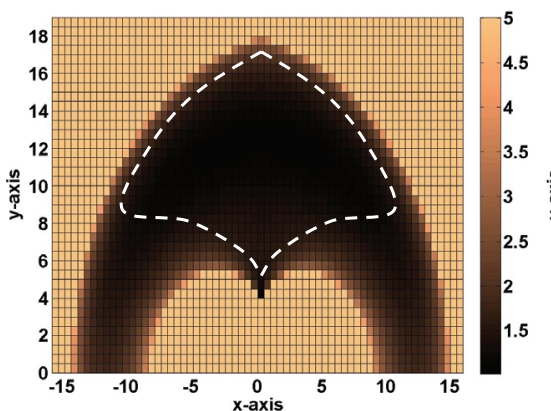

(a)

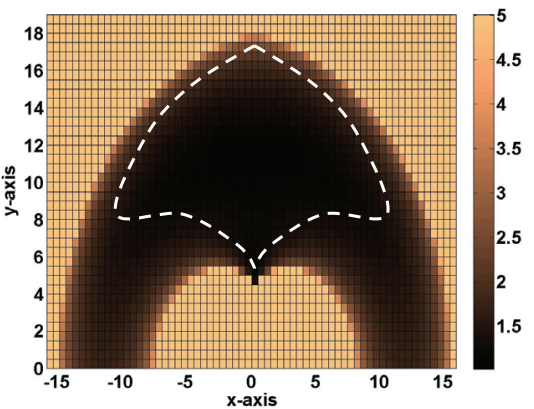

(b)

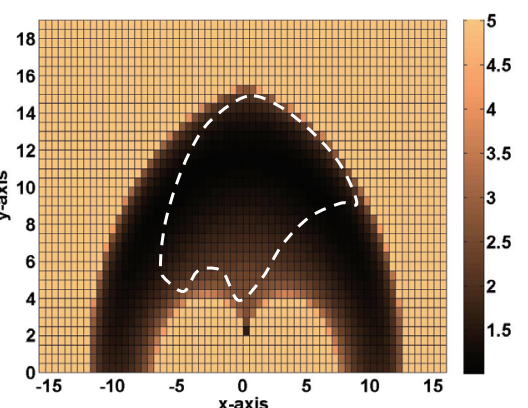

(c)

Fig. 5. Manipulability and feasible workspace without link collision (dotted line); (a) G10, (b) G6, (c) P10.

assemblability had to be carefully considered. As realized, the tactor has the workspace shown with dotted lines in Fig. 5. The workspace is $10 \mathrm{~mm}$ in the $y$-axis and, at maximum, $12 \mathrm{~mm}$ in the $x$-axis, satisfying the design specifications discussed in section II. The distorted workspace of P10 in Fig. 5 is resulted from the intentional mechanical joint limits to avoid the kinematic singularities.

\section{B. Actuator Selection}

A number of different types of actuators were considered. For instance, ultrasonic motors were carefully considered since they can be designed to produce high torque and low speed without reductions. However, to date, this type of actuator would not meet the performance requirements due to poor frequency response and an inability to achieve high accelerations over a sufficiently long period of time.

It became apparent that rotary electromagnetic motors and gearboxes would be the best choice, at least for a first generation design. For the P10 and G10 tactors, we selected a $10 \mathrm{~mm}$ diameter DC brushed motor (256105, Maxon Motor), which has a peak mechanical power output of 1.5 watts. We also selected a 16 : 1 planetary gearhead (218416, Maxon Motor) resulting in a maximum torque of $51.8 \mathrm{mNm}$ after reduction.

The motors have relatively high no-load speeds of $781 \mathrm{rpm}$ for G10 and P10 and 833 rpm for G6 after gear reduction. This corresponds to 1 to $10 \mathrm{~m} / \mathrm{sec}$ across the whole workspace, so there is no problem meeting the speed requirements. Although this motor meets our specification, there are good reasons to continue exploring actuator alternatives. The $10 \mathrm{~mm}$ diameter of the motor, while small, is still large enough to seriously compromise the number of tactors that might be used, and to cause unsightly bulges.

To explore the possibility of creating a smaller tactor, we designed the G6 tactor using the $6 \mathrm{~mm}$ diameter DC brushless motor (250101, Maxon Motor), which has a peak mechanical power output of 1.2 watts. A maximum torque of $22.9 \mathrm{mNm}$ is available after reduction with a $57: 1$ planetary gearhead (199689, Maxon Motor). ${ }^{3}$ In addition to being smaller, this actuator has the benefit of being brushless. Brushes are a source of electrical noise and wear. On the other hand, there are some potential disadvantages associated with the larger gear ratio including decreased backdriveability and increased backlash. Both of these factors are known to affect the dynamic performance of robotic systems [42], [43]. Another potential disadvantage of the smaller motor is that the connectors to its optical encoder are prone to failure. Nonetheless, these motors have worked well in tests to date.

Fig. 6 shows the torques required to create $9.0 \mathrm{~N}$ force

${ }^{3}$ Due to the gear box maximum load capability and the backlash issues, $57: 1$ was the maximum acceptable reduction ratio. 


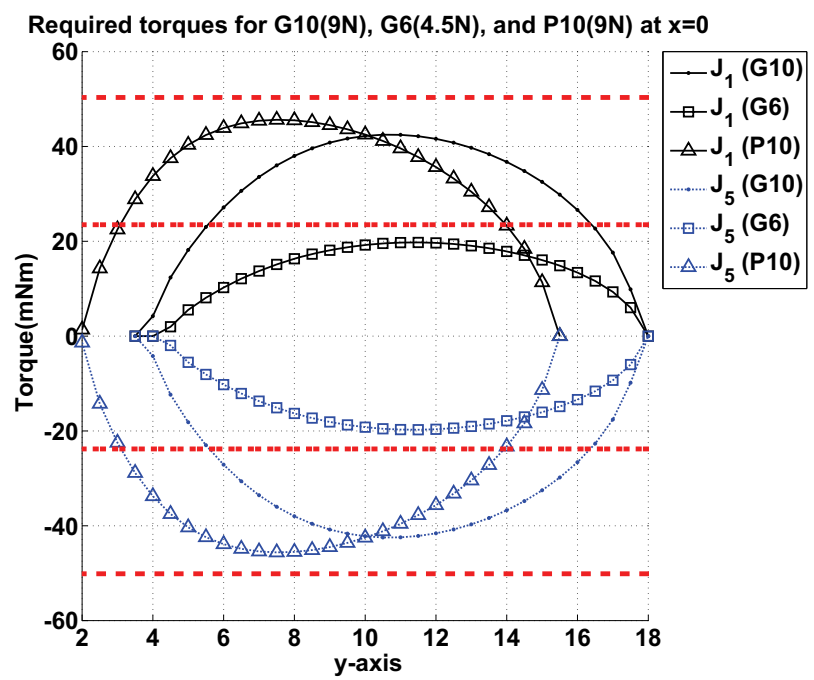

(a)

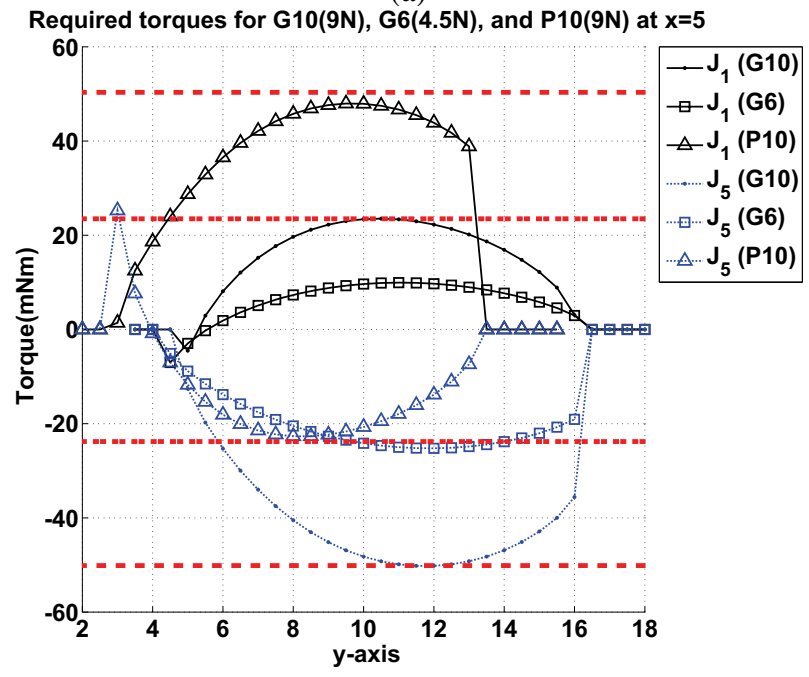

(b)

Fig. 6. Required torques to create $9.0 \mathrm{~N}$ force for $\mathrm{P} 10$ and $\mathrm{G} 10,4.5 \mathrm{~N}$ for G6 along $y$ direction: (a) when $x=0$, (b) when $x=5$. Dotted horizontal lines represent maximum torque of the actuator for G6 $(22.9 \mathrm{mNm})$. Dashdotted lines represent maximum torque of the actuator for G10 and P10 (51.8 $\mathrm{mNm})$.

along the $y$-axis when $x=0$ and when $x=5$. The results indicate that the necessary torques are less than the maximum torque of the selected actuator, $51.8 \mathrm{mNm}$, throughout most of the workspace for P10 and G10. However, for G6, the 9 $\mathrm{N}$ specification is too high. Thus, Fig. 6 shows the torque required to create $4.5 \mathrm{~N}$ of $y$-axis force. This lower force specification seems like a reasonable trade-off for a smaller haptic display, especially since the original specification of 9 $\mathrm{N}$ was quite aggressive, above the pain threshold for two TR patients.

\section{Force Sensors}

Force sensors are required for the closed loop control of contact, pressure, vibration, and shear force. The end-effector is an ideal sensing location, but in the case of P10, a thermoelectric device is also placed on that location for thermal

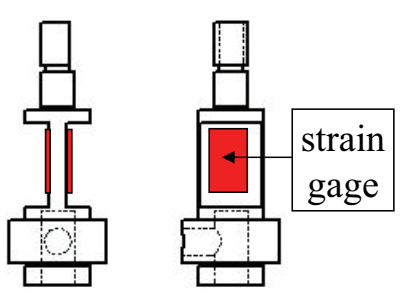

(a)
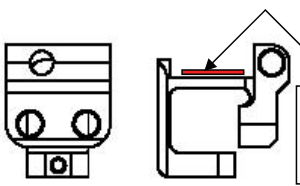

(c)

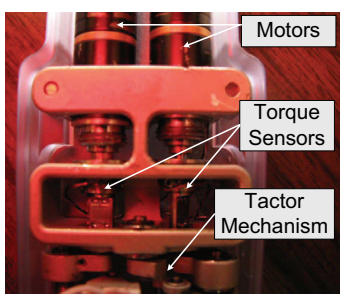

(b)

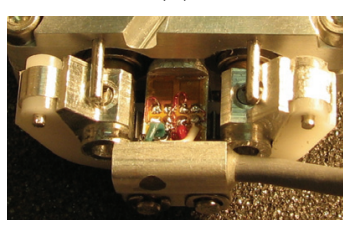

(d)
Fig. 7. Prototype of the torque sensor and the force sensor: (a) Schematic drawing of the torque sensor for $\mathrm{P} 10$, (b) the torque sensors implemented in $\mathrm{P} 10$, (c) schematic drawing of the forces sensor for G6 and G10, (d) the force sensor implemented in G10.

display. The associated thermal gradients can be expected to cause significant sensor drift. An alternative is to measure the torques output by the motor/gearboxes and compute the force at the tactor head using the conventional torque to force mapping. However, since, to the best of the authors' knowledge, there are no commercially available torque sensors that satisfy the design constraints/specifications, we set out to design custom sensors.

The smallest metal foil strain gage that we were able to find for shear stress was the EA-06-062TV-350 (Micromeasurement), which limited the size of the sensing parts to 3.87 $\mathrm{mm} \times 5.10 \mathrm{~mm}$ for 4 double faced strain gages. Titanium is used to maximize the yield strength as well as the sensitivity of the torque sensor. Using Solidworks COSMOS Tools, the flexure was sized to ensure that the maximum stress on the torque sensor is less than $138 \mathrm{kPa}$ under $50.0 \mathrm{mNm}$ applied torque. Since titanium has a $0.2 \%$ yield strength of $1,034 \mathrm{kPa}$, we are confident that no actual damage will occur in operation. Fig. 7(a) shows the schematic drawing of the torque sensor as manufactured by Sensing System Co.( [44]), which has a sensitivity of $0.826 \mathrm{mV} / \mathrm{V}$, a maximum torque capacity of 50.0 $\mathrm{mNm}$, and a combined cross talk error of $0.071 \%$. This torque sensor is implemented for P10 as shown in Fig. 7(b).

On the other hand, if the task does not require a thermal display, the best location for measuring force is the endeffector. Thus, a miniature force sensor has been developed and mounted on the end-effectors of G10 and G6. The same small metal foil gages mentioned above were used for this sensor. Fig. 7(d) shows the force sensor as manufactured by Sensing System Co. which has a sensitivity $1.4 \mathrm{mV} / \mathrm{V}$, a maximum force capacity of $8.9 \mathrm{~N}$, and a combined cross talk error of $0.066 \%$. Note that this is a single-axis sensor, meaning that it measures forces normal to the end-effector. In operation, these are assumed to be $y$-axis forces even though the endeffector (tactor head) may tilt. 


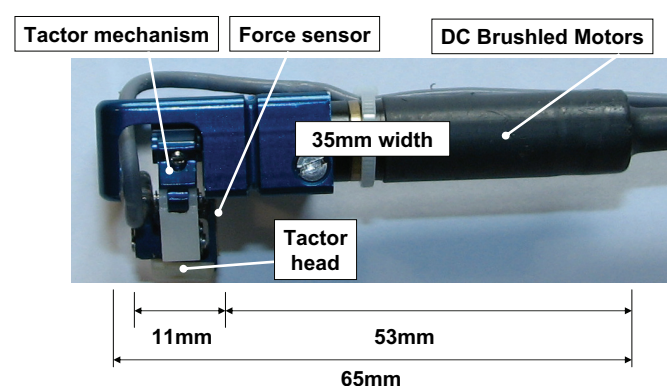

(a)

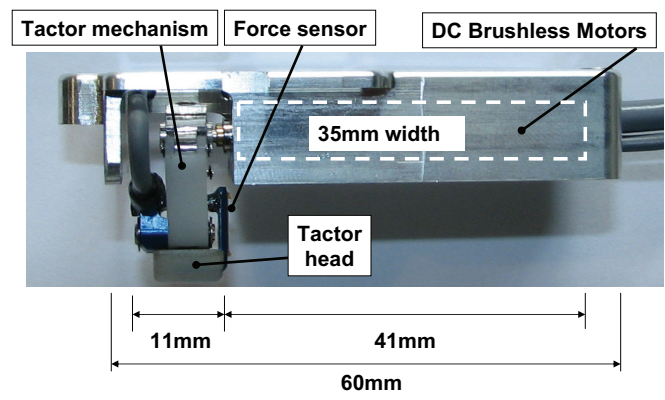

(b)

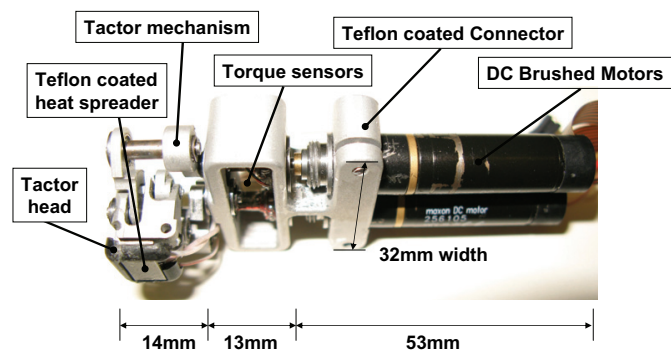

(c)

Fig. 8. Dimensions and masses of the components of the tactors: (a) G10 with the mass of $41 \mathrm{~g}$ (total mass) $=4 \mathrm{~g}$ (mechanism) $+1 \mathrm{~g}$ (force/torque sensors) $+36 \mathrm{~g}$ (actuators). (b) G6 with the mass of $16 \mathrm{~g}$ (total mass) $=3 \mathrm{~g}$ (mechanism) $+1 \mathrm{~g}$ (force/torque sensors) $+12 g$ (actuators). (c) P10 with the mass of $45 \mathrm{~g}$ (total mass) $=7 \mathrm{~g}$ (mechanism) $+2 \mathrm{~g}$ (force/torque sensors) $+36 \mathrm{~g}$ (actuators).

\section{Mass and Dimension}

Fig. 8 describes the dimensions and the masses of the tactors. The masses of G6, G10, and P10 are 16 grams, 41 grams, and 45 grams, respectively. The lengths of G6, G10, and P10 are $60 \mathrm{~mm}, 65 \mathrm{~mm}$, and $80 \mathrm{~mm}$. Since the actuator is the dominant factor to determine the total mass and size, it is natural that G6 is the most compact design. P10 is the longest design since torque sensors are implemented between the actuator and the mechanism while the others use a force sensor at the end-effector.

\section{Performance Comparison}

The three tactors have been developed with various combinations of mechanisms and actuators and placing emphasis on different design considerations. This section compares the performance of the tactors in terms of one, open loop

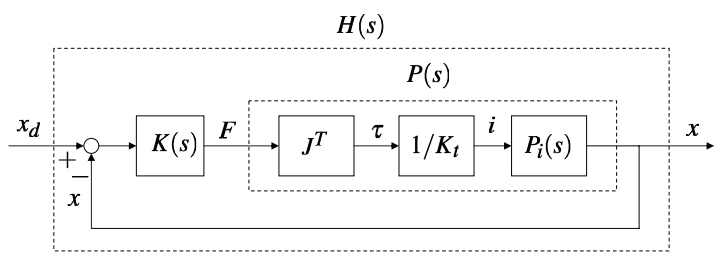

Fig. 9. Block diagram for open loop frequency response in free motion.

frequency response in free motion; two, closed loop force frequency response with constrained motion; and three, closed loop tapping response with constrained motion.

\section{A. Open loop frequency response in free motion}

From the open loop frequency response in free motion, we can estimate the dynamics of the tactor mechanisms combined with the actuators and gearboxes. Though this does not directly show the haptic display performance on a patient's body, it is a meaningful baseline for understanding the intrinsic dynamics of each device. The force-to-position frequency response is useful to estimate the performance in high frequency vibration since motion amplitude is one of the dominant factors relating to vibration perception.

Fig. 9 shows the control block diagram used to measure the open loop frequency response. ${ }^{4} \boldsymbol{x}_{d}\left(x_{d}, y_{d}\right)$ and $\boldsymbol{x}(x, y)$ are the desired position and the actual position of the end-effector. From the controller $\boldsymbol{K}(s)$, the applied force $\boldsymbol{F}$ is calculated. Motor torque, $\boldsymbol{\tau}$, can be calculated from the Jacobian, $\boldsymbol{J}$. $K_{t}$, the motor constant, maps current, $\boldsymbol{i}$, to torque. Here, we use the inverse relationship. $\boldsymbol{P}_{i}(s)$ represents the dynamics of the haptic devices from the current input to the position output. $\boldsymbol{P}(s)$ is the force-to-position open loop linear dynamic model to be calculated. Although $\boldsymbol{P}(s)$ can in principle be calculated from direct measurements of position responding to the input, these haptic devices have no intrinsic spring return to an equilibrium configuration, causing them to drift when driven with a persistent zero mean signal such as a sinusoid or white noise. To eliminate this drift, we introduced small gain PD controller, $\boldsymbol{K}(s)$ as well as the excitation signal.

$$
\begin{aligned}
\boldsymbol{x}(t) & =\boldsymbol{P}(s) \boldsymbol{F}(t) \\
\boldsymbol{F}(t) & =K_{p}\left(\boldsymbol{x}_{d}(t)-\boldsymbol{x}(t)\right)-K_{d} \dot{\boldsymbol{x}}(t),
\end{aligned}
$$

where $K_{p}$ and $K_{d}$ are proportional and derivative controller gains.

Since the tactor is basically a multi-DOF non-linear manipulator, we can expect that the frequency response will depend on its pose and direction of motion. It is nonetheless valuable to find frequency response in a few nominal configurations in order to identify resonance and other dynamic characteristics. Here we present data for two conditions: one, an $x$-direction

${ }^{4}$ The experiments described in this paper have been performed using a PC 104 format computer running the MATLAB xPC real time operating system with a $2 \mathrm{kHz}$ sampling frequency for $\mathrm{P} 10$; and using a QNX real time operating system through CAN communication with a $2 \mathrm{kHz}$ sampling frequency for G10 and G6. 
excitation, and the other a $y$-direction excitation. For the $x$ direction, $\boldsymbol{x}_{d}(t)=[2 \sin (w t), 5]^{T}(\mathrm{~mm})$. For the $y$-direction, $\boldsymbol{x}_{d}(t)=[0,2 \sin (w t)+8]^{T}(\mathrm{~mm})$.

The open loop transfer function $P(s)$ that we wish to find relates to the closed loop transfer function $H(s)$ as follows:

$H(s)=P(s) K_{p} /\left(1+P(s) K_{p}+P(s) K_{d} s\right)=X(s) / X_{d}(s)$,

where $X_{d}(s)$ and $X(s)$ are the Laplace transforms of $x_{d}(t)$ and $x(t)$, respectively. We use the inverse of this equation to solve for $P(s)^{5}$ :

$$
P(s)=H(s) /\left(K_{p}-H(s) K_{p}-H(s) K_{d} s\right) .
$$

Fig. 10(a) shows the frequency response of the tactors, i.e., $\boldsymbol{P}(s)$. Up to $30 \mathrm{~Hz}$, encoder signals are used to calculate the motion of the end effector. Between $30 \mathrm{~Hz}$ to $400 \mathrm{~Hz}$, an accelerometer (LIS3L06AL, STMicroelectronics) mounted on the tactor head is used to provide a direct measurement of motion. P10 has higher response magnitude than G10 and G6, probably due to its ball-bearing joints and the lack of a gear constraint. At low frequency (less than $3 \mathrm{~Hz}$ ) G6 has lower magnitude than G10. It seems likely that this results from the added friction associated with a high gear ratio reduction. We didn't plot the response beyond $35 \mathrm{~Hz}$ for G6 since below $-30 \mathrm{~dB}(0.05 \mathrm{~mm})$ is in the range of backlash of the gear box of G6.

Fig. 10(b) shows the frequency responses scaled by the motors' maximum continuous torque output, which lets us treat the responses as maximum position amplitude plots. Also shown on the same axes are vibration detection thresholds for fingers and forearms as measured by Bolanowski et al. [45], [46]. P10 is capable of generating vibrations greater than the thresholds up to $250 \mathrm{~Hz}$. Above $250 \mathrm{~Hz}$, the magnitude of the vibration is less than the threshold of the forearm while still higher than that of hand. G10 is capable of generating vibrations greater than the thresholds up to $100 \mathrm{~Hz}$. Above 100 $\mathrm{Hz}$, the magnitude is less than the threshold of the forearm but higher than that of hand. However, we experienced that while not much of the high frequency may be propagated through the gearbox, it gets to the skin anyway through the motor mounting. This is useful because the Pacinian corpuscles, which are responsible for high-frequency vibration response, have a broad receptive field and are not directionally sensitive. Of course, it remains to be established that Pacinian corpuscles are responsible for vibration sensing in reinnervated skin, and that reinnervated skin has vibration sensitivity comparable to that of intact finger or forearm.

\section{B. Closed Loop Force Response}

As described above, we tend to think of the high frequency behavior of the tactors in terms of vibration amplitude. At lower frequencies, however, we think of the tactors as force

\footnotetext{
${ }^{5} H(s)$ and $P(s)$ were calculated from a MATLAB program to compute the discrete fourier transform based on the sinusoidal data sets of $x_{d}(t)$ and $x(t)$ containing time-domain data longer than 10 periods at the pre-defined frequencies. The frequencies are 30 points equally distributed from $1 \mathrm{~Hz}$ to $500 \mathrm{~Hz}$.
}

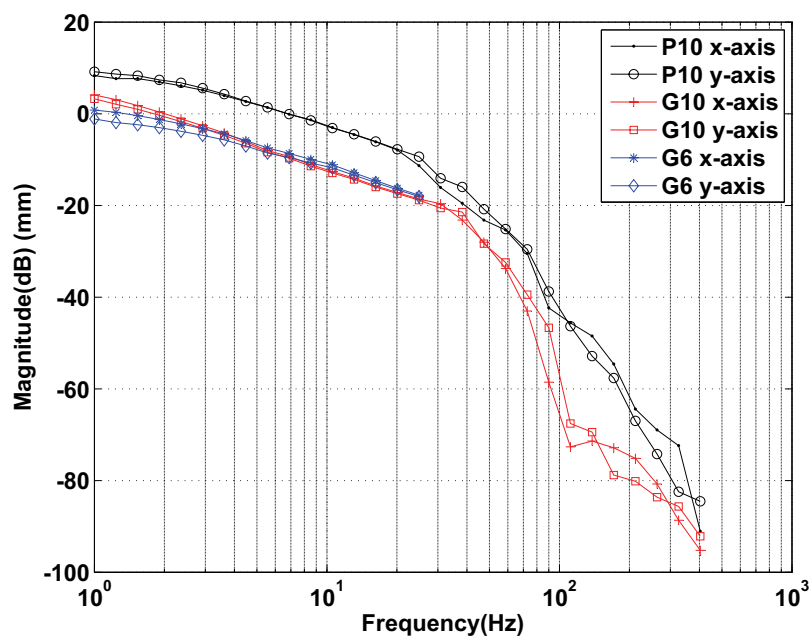

(a)

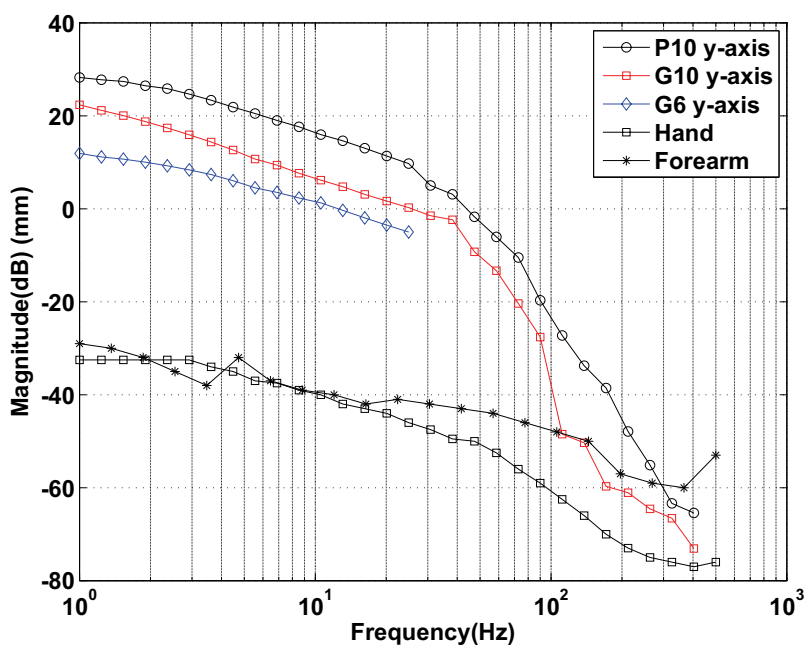

(b)

Fig. 10. (a) Frequency response in free motion, (b) Vibration detection threshold of hand, forearm, and abdomen [45], [46] vs. frequency response of the tactors scaled by the motors' maximum continuous torque output $(24.64 \mathrm{mNm}$ for P10 and G10, $13.22 \mathrm{mNm}$ for G6). x-direction at $x=-3.0 \mathrm{~mm}$ and $y=8.0 \mathrm{~mm}$ and y-direction at $x=-3.0 \mathrm{~mm}$ and $y=5.0 \mathrm{~mm}$.

sources. As such, force or torque feedback is used to implement closed loop force control. Experiments were performed to measure the closed loop frequency response of the tactors while pressing against a compliant silicone pad.

Fig. 11 shows the control block diagram used to measure the closed loop force response in constrained motion. $\boldsymbol{F}_{d}\left(F_{x d}, F_{y d}\right)$ and $\boldsymbol{F}_{s}\left(F_{s d}, F_{s d}\right)$ are the desired force and the measured force. The desired position is calculated as $\boldsymbol{x}_{d}=K_{i} \int\left(\boldsymbol{F}_{d}-\boldsymbol{F}_{s}\right) d t+K_{p} \boldsymbol{F}_{s}$. IK denotes the inverse kinematics. $q_{d}$ and $q$ are the desired angle and the actual angle of the haptic device. $\boldsymbol{K}(s)$ is a controller and $\boldsymbol{P}(s)$ is the dynamics of the tactor.

The experimental results were taken with the tactor firmly clamped to a tabletop. A sticky and flexible silicon pad (feeling similar to skin) was placed below the end effector. Sinusoidal commands were input to measure frequency response, and 


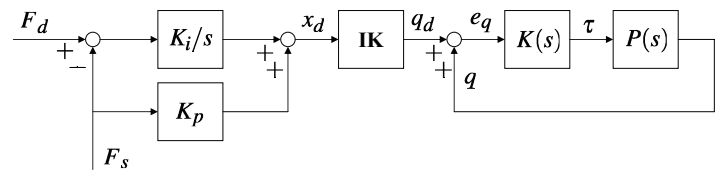

Fig. 11. Block diagram for closed loop force response in constrained motion.

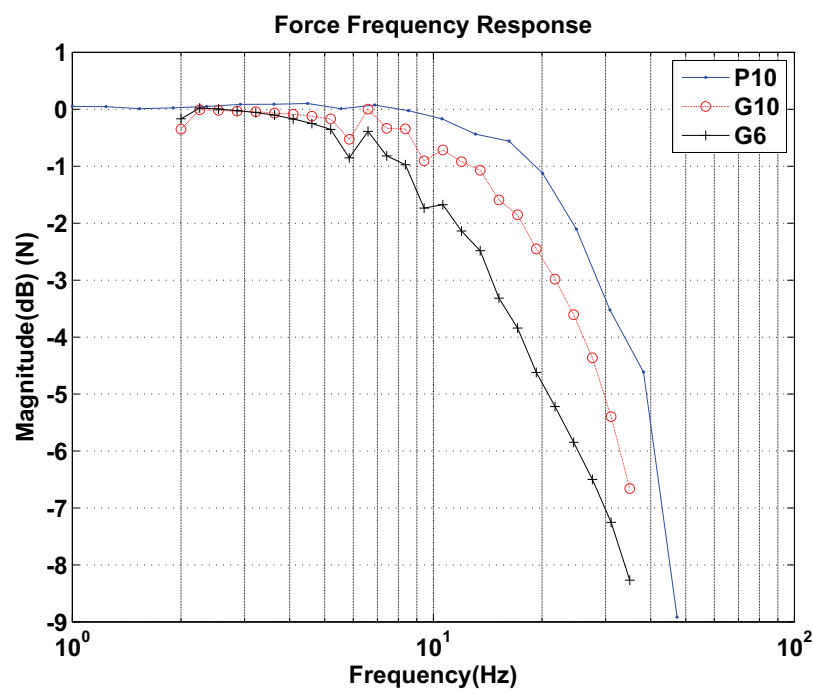

Fig. 12. Force frequency response in constrained motion.

two types of pulse commands were input to measure tapping response. In the experiments, we assumed that the controller was well tuned to guarantee the best performance under the given tasks. $\boldsymbol{F}_{d}$ for the closed loop force response was modeled as:

$$
\boldsymbol{F}_{d}=A_{0}+A \sin (\omega t)
$$

where $A_{0}=1.5(\mathrm{~N})$ and $A=1.0(\mathrm{~N})$.

The experimental results are plotted in Fig. 12. The cut-off frequencies of G6, G10, and P10 are $15 \mathrm{~Hz}, 21 \mathrm{~Hz}$, and 29 $\mathrm{Hz}$, respectively. The differences are attributable to backlash, mechanism friction (greater for the geared mechanisms), and apparent inertia (greatest for G6 with its higher gear ratio).

\section{Tapping Response in Constrained Motion}

As explained in section II, contact has top priority among the sensations to be restored. In order to compare the ability of the three tactors to deliver a crisp contact sensation, the response to tapping commands was compared using the same controller used in the closed loop force response. Two types of tapping command were considered: one, a continuous (smooth) input, and the other, a discontinuous (sharp cornered) inputs. For continuous tapping, the desired command is:

$f_{d}(t)= \begin{cases}A_{0}+\frac{A}{2}\left\{1-\cos \left(\frac{2 \pi\left(t-t_{0}\right)}{w}\right)\right\} & \text { if } t_{0} \leq t \leq t_{0}+w \\ A_{0} & \text { else. }\end{cases}$

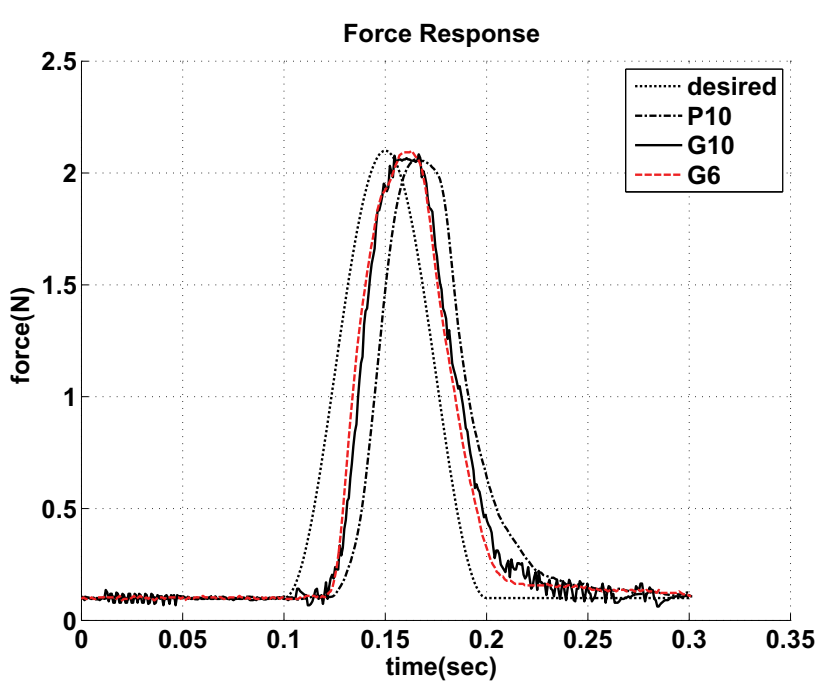

(a)

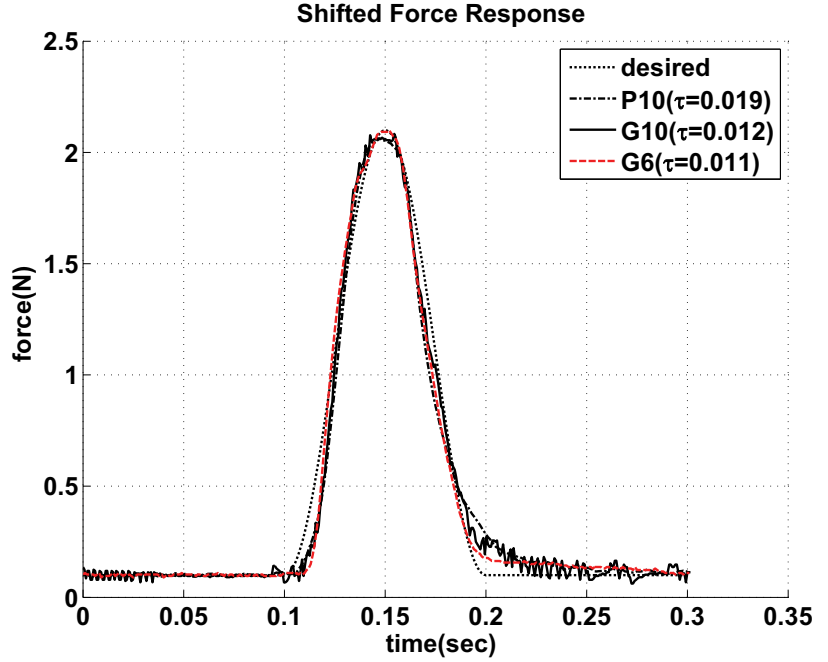

(b)

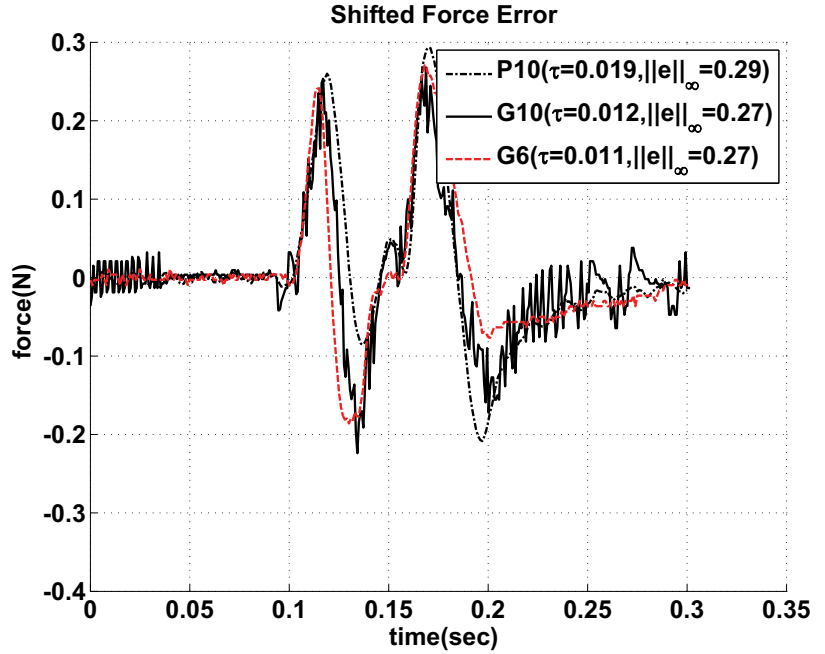

(c)

Fig. 13. Force response for the continuous tapping command: (a) Original experimental results, (b) Shifted data minimizing given error norm, (c) errors of the shifted data.

For discontinuous tapping, the desired command is: 


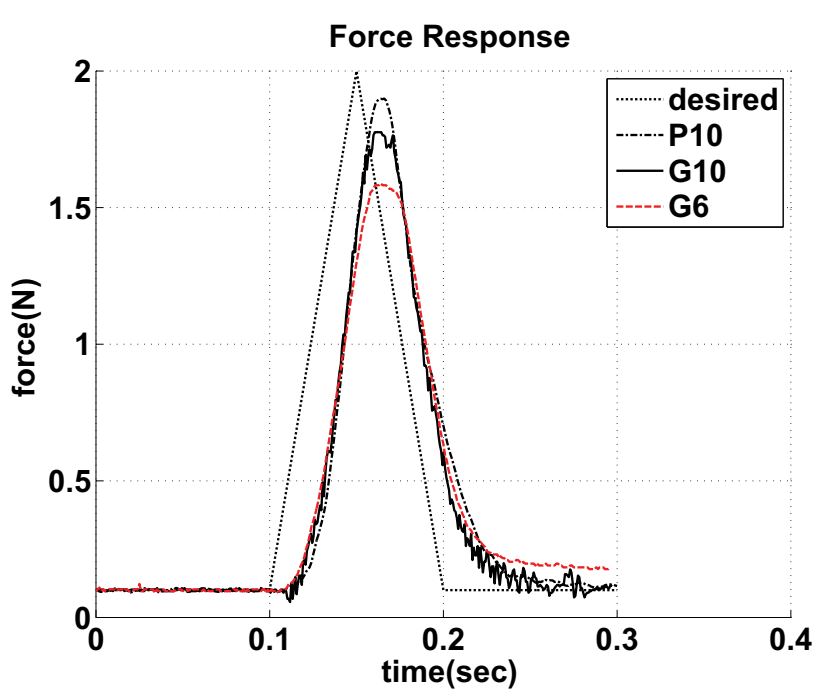

(a)

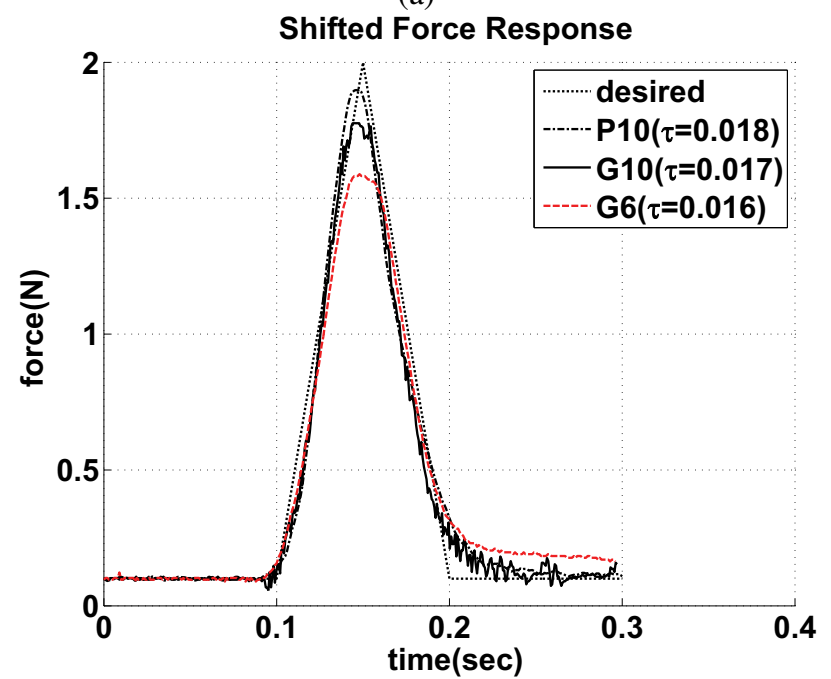

(b)

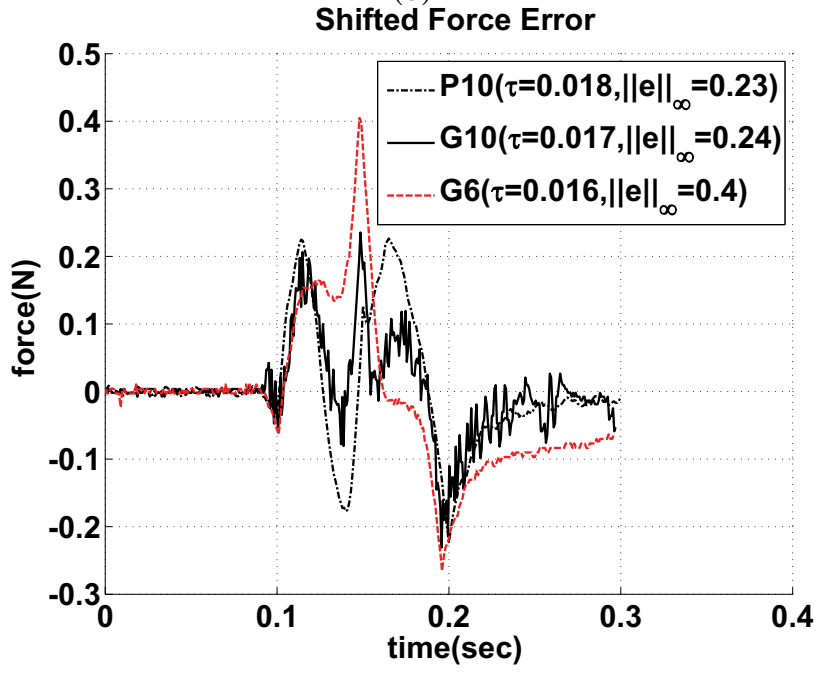

(c)

Fig. 14. Force response for the discontinuous tapping command: (a) Original experimental results, (b) Shifted data minimizing given error norm, (c) errors of the shifted data.

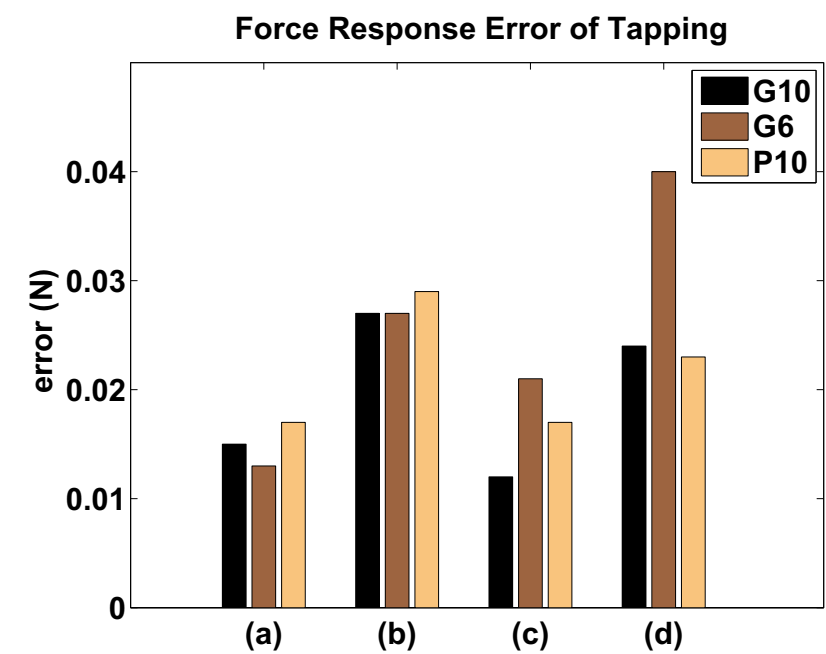

Fig. 15. Force response error for tapping: (a) $\int e d t \times 10$ of continuous tapping, (b) $|e|_{\infty}$ of continuous tapping, (c) $\int e d t \times 10$ of discontinuous tapping, (d) $|e|_{\infty}$ of discontinuous tapping.

$$
f_{d}(t)= \begin{cases}A_{0}+2 A \frac{t-t_{0}}{w} & \text { if } t_{0}<t<t_{0}+w / 2 \\ A_{0}-2 A \frac{t-t_{0}-w}{w} & \text { if } t_{0}+\frac{w}{2}<t<t_{0}+w, \\ A_{0} & \text { else. }\end{cases}
$$

In this experiment, for both commands, $A_{0}=0.1, A=2.0$, $w=0.1$, and $t_{0}>w$.

Fig. 13(a) and Fig. 14(a) show the desired force command and the responses. In order to compare the responses quantitatively without considering phase delay, the response data were shifted to minimize the following cost function (shifted data are shown in Fig. 13(b) and Fig. 14(b)):

$$
\left.\tau=\left\{\tau \mid \min _{\tau \in R} g(e(t, \tau)), e(t, \tau)=f_{d}(t)-f_{s}(t-\tau)\right)\right\} .
$$

Fig. 13(c) and Fig. 14(c) show the error between $f_{d}(t)$ and $f_{s}(t-\tau)$ when $g(e)=\|e\|_{\infty}$ or $g(e)=\int e d t$. As shown in Fig. 15, the errors are not significantly different when the command is continuous and smooth. However, if the tapping becomes more discontinuous, G6 shows significantly more error than others. This again results from the high gear ratio of the actuator. P10 shows slightly more error and longer $\boldsymbol{\tau}$ than G6 when $g(e)=\int e d t$. This results from the higher inertia of the 6-bar skewed parallelogram mechanism (7 grams) compared to the 6-bar gear constraint mechanism (4 grams).

\section{CONCLUding REMARKS}

We have developed three different versions of a multifunction tactor that is able to provide contact, pressure, vibration, shear force, and temperature feedback to upper extremity amputees, especially those who have undergone TR surgery. We proposed two kinematic designs of closed loop 6-bar mechanisms, one with gear constraints, and one with a skewed parallelogram linkage. Both designs provide normal and shear motion, and the parallelogram linkage further keeps the tactor head parallel to the skin surface. The tactors also achieve the 


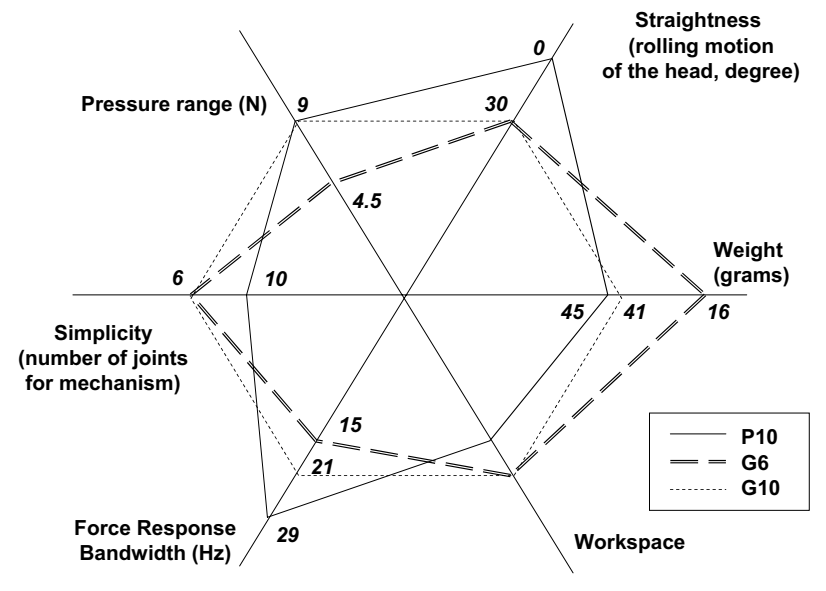

Fig. 16. Comparison of the proposed haptic devices.

following characteristics: $i$ ) workspace maximization subject to good manipulability; $i$ ) size minimization through careful linkage design and close-tolerance machining; iii) a unique bearing configuration to avoid over-constraint; $i v$ ) customized force and torque sensors for closed loop force control. All three are small and light weight enough to be wearable devices. We have also presented data illustrating good bandwidth and excellent force tracking performance satisfying the performance and design specifications described in Table I.

Fig. 16 shows the trade-offs of the design and the performance of the tactors. G10 has little backlash, a simple mechanism, and meets the performance requirements, but it does not keep the tactor head parallel to skin during side-toside motion. The force response bandwidth is also less than P10. G6 has simple mechanism like G10, is very light weight, and is smaller in size than the other two. But the high gear reduction and the gear constraints reduce the bandwidth. It does not meet the pressure specification due to its lower power actuator, but this seems like a reasonable trade-off since the original specification was quite aggressive. P10 has a complex mechanism in order to keep the tactor head parallel to skin at any pose while it meets the performance requirements. Due to the large number of joints and the difficult preloading such tiny bearings, the final prototype has a slight wobble when not preloaded. This is not a significant problem since the tactor is always used under some amount of preload. P10 has very good bandwidth for force response in constrained motion as well as in free motion. Understanding these trade-offs should help to guide design decisions depending on each patient's needs and situation.

All three tactors have been used in preliminary tests with two TR patients, and the results are very encouraging. In the experiments, we mounted G10 on a TR patient's reinnervated skin in a spot that corresponded to the palm of the hand. A fingertip sensor consisting of a 3 -axis force sensor and a 3-axis accelerometer was mounted on the prosthetic hand. Modalities were correctly perceived (i.e., pressure is perceived as pressure, vibration as vibration, and contact as contact). For the vibration test, the blindfolded TR patients were asked to discriminate between two surfaces of the same material, with the surfaces differing in roughness. The materials used were sand paper, Teflon, and ribbon cable. 8 trials were performed for each material, i.e., 24 total trials per subject. One TR patient was able to distinguish the surface roughnesses with 91 percent accuracy, and the other TR patient performed at 96 percent accuracy. One subject discriminated the surface roughness of sand paper, Teflon, and ribbon cable with 6 out of 8,8 out of 8 , and 8 out of 8 correct, respectively. The other subject discriminated the surface roughness of sand paper, Teflon, and ribbon cable with 8 out of 8,7 out of 8 , and 8 out of 8 correct, respectively. Also, using the shear display, they were able to feel surfaces with different friction coefficients. One of the patients described the sensation as a rocking motion projected to her missing hand (although it is not yet clear how well patients can discriminate pressure from shear). Formal tests are ongoing and will be reported in the future. We are also making plans for a take home testing of the tactors to get patient impressions following extended use. In a take home test, the patients experience the device in their daily lives and give feedback for improvement. This type of testing will also highlight unexpected usages in various environments.

Even in the absence of additional patient tests, it is clear that there are improvements to be made in the tactor design. For instance, it is desirable to shrink the size of the tactor even further. The tactor must compete with EMG electrodes and socket components for space on the patient's skin. Thus, smaller is always better. While we may be able to accomplish this by using even smaller motors, another option is to remove functionality. The TR patients with whom we work have informally prioritized the desired sensations from most to least important: touch (contact), pressure, temperature, vibration and shear. Contact and pressure are most important, and can be conveyed using a much simpler single-DOF design. An interesting direction that we will pursue in the future is the use of several reduced-functionality tactors, each communicating with sensors on a different part of the prosthetic hand. An important research question is whether multiple tactors will provide more useful information, or simply more confusion.

\section{ACKNOWLEDGMENT}

We wish to thank Todd A. Kuiken, Laura A. Miller, Blair Lock, and Paul D. Marasco for their assistance and advice. We wish to acknowledge Prof. Ronald Johansson, members of Prof. Paolo Dario's Cyberhand group and Prof. Allison Okamura for discussing with us the prioritization of haptic sensations.

\section{REFERENCES}

[1] P. Bach-y-Rita, Tactile Sensory Substitution Studies. Ann. N.Y. Acad Sci.: The Coevolution of Human Potential and Converging Technologies, 2004, vol. 1013.

[2] G. Shannon, "A comparison of alternative means of providing sensory feedback on upper limb prostheses," Medical and biological engineering and computing, vol. 14, pp. 289-294, 1976.

[3] P. Nohama, A. Lopes, and A. Cliquet, "Electrotactile stimulator for artificial proprioception," Artificial Organs, vol. 19, no. 3, pp. 225-230, 1995.

[4] G. Wang, X. Zhang, J. Zhang, and W. Gruver, "Gripping force sensory feedback for a myoelectrically controlled forearm prosthesis," in IEEE International Conference on Intelligent Systems for the 21st Century, Vancouver, BC, 1995, pp. 501-504. 
[5] S. G. Meek, S. C. Jacobsen, and P. P. Goulding, "Extended physiologic taction: Design and evaluation of a proportional force feedback system," Journal of Rehabilitation Research and Development, vol. 26, no. 3, pp. 53-62, 1989.

[6] D. S. Childress, "Closed-loop control in prosthetic systems - historical perspective," Annals of biomedical engineering, vol. 8, no. 4-6, pp. 293 303, 1980.

[7] C. A. Phillips, "Sensory feedback-control of upper-extremity and lowerextremity motor prostheses," Critical reviews in biomedical engineering, vol. 16, no. 2, pp. 105-140, 1998.

[8] P. Patterson and J. Katz, "Design and evaluation of a sensory feedbacksystem that provides grasping pressure in a myoelectric hand," Bulletin of prosthetics research, vol. 29, no. 1, pp. 1-8, 1992.

[9] T. A. Kuiken, L. A. Miller, R. D. Lipschutz, K. A. Stubblefield, and G. A. Dumanian, "Prosthetic command signals following targeted hyperreinnervation nerve transfer surgery," in Proc. of IEEE Enginerring in Medicine and Biology 27th Annual Conference, 2005.

[10] T. A. Kuiken, G. A. Dumanian, R. D. Lipschutz, L. A. Miller, and K. A. Stubblefield, "Targeted reinnervation for enhanced prosthetic arm function in a woman with a proximal amputation: a case study," vol 369, pp. 371-380, Feb. 2007.

[11] T. A. Kuiken, L. A. Miller, R. D. Lipschutz, B. A. Lock, K. S. eld, P. D. Marasco, P. Zhou, and G. A. Dumanian, "The use of targeted muscle reinnervation for improved myoelectric prosthesis control in a bilateral shoulder disarticulation amputee," Prosthetics and orthotics international, vol. 28(3), pp. 245-253, Dec. 2004.

[12] T. A. Kuiken, "Consideration of nerve-muscle grafts to improve the control of artificial arms," Journal of Technology and Disability, vol. 15 pp. 105-111, 2003

[13] L. Beccai, S. Roccella, L. Ascari, P. Valdastri, A. Sieber, M. C. Carrozza, and P. Dario, "Development and experimental analysis of a soft compliant tactile microsensor for anthropomorphic artificial hand," IEEE/ASME Transactions on Mechatronics, vol. 13, no. 2, pp. 158-168, 2008 .

[14] L. Zollo, S. Roccella, E. Guglielmelli, M. C. Carrozza, and P. Dario, "Biomechatronic design and control of an anthropomorphic artificial hand for prosthetic and robotic applications," IEEE/ASME Transactions on Mechatronics, vol. 12, no. 4, pp. 418-429, 2007

[15] T. A. Kuiken, P. D. Marasco, B. A. Lock, R. N. Harden, and J. P. A Dewald, "Redirection of cutaneous sensation from the hand to the chest skin of human amputees with targeted reinnervation," Proceedings of the National Academy of Sciences, vol. 104, no. 50, pp. 20061-20066, December 2007.

[16] H.-N. Ho and L. A. Jones, "Thermal model for hand-object interactions," in Proc. Symposium on Haptic Interface for Virtual Environment and Teleoperator Systems, 2006

[17] A. Yamamoto, B. Cros, H. Hashimoto, and T. Higuchi, "Control of thermal tactile display based on prediction of contact temperature," in Proc. IEEE international conference on robotics and automation, 2004.

[18] K. Kim, J. E. Colgate, and M. A. Peshkin, "On the design of a thermal display for upper extremity prosthetics," in Haptic Interfaces for Virtual Environment and Teleoperator Systems 2008, 2008, pp. 413-419.

[19] T. A. Kuiken, J. W. Sensigner, T. R. Farrell, and R. ff, "Phantom limb sensory feedback through nerve transfer surgery," in American Academy of Orthotists and Prosthetists Scientific Symposium, 2007.

[20] H. Kajimoto, N. Kawakami, T. Maeda, and S. Tachi, "Tactile feeling display using functional electrical stimulation," in The Ninth International Conference on Artificial reality and Telexistence (ICAT'99), 1999.

[21] T. Iwamoto and H. Shinoda, "Finger ring tactile interface based on propagating elastic waves on human fingers," in Proceedings of the Second Joint EuroHaptics Conference and Symposium on Haptic Interfaces for Virtual Environment and Teleoperator Systems, 2007, pp. 145-150.

[22] N. S. Methil, Y. Shen, D. Zhu, C. A. Pmeroy, and R. Mukherjee, "Development of supermedia interface for telediagnostics of breast pathology," in Proceedings of the 2006 IEEE International Conference on Robotics and Automation, Orland, Florida, 2006, pp. 3911-3916.

[23] K. Sato, H. Kajimoto, N. Kawakami, and S. tachi, "Improvement of shape distinction by kinesthetic-tactile integration," in Proceedings of the Second Joint EuroHaptics Conference and Symposium on Haptic Interfaces for Virtual Environment and Teleoperator Systems, 2007, pp 391-396.

[24] H. Kajimoto, N. Kawakami, and S. Tachi, "Psychophysical evaluation of receptor selectivity in electro-tactile display," in 13th Int. Sympo. on Measurement and Control in Robotics (ISMCR), Madrid, Spain, 2003.

[25] S. Kuroki, H. Kajimoto, H. Nii, N. Kawakami, and S. Tachi, "Proposal for tactile sense presentation that combines electrical and mechanical stimulus," in Proceedings of the Second Joint EuroHaptics Conference and Symposium on Haptic Interfaces for Virtual Environment and Teleoperator Systems, 2007, pp. 121-126.

[26] Q. Wang and V. Hayward, "Compact, portable, modular, highperformance, distributed tactile transducer device based on lateral skin deformation," in 2006 Symposium on Haptic Interface for Virtual Environment and Teleoperator Systems IEEE VR, 2006, pp. 67-72.

[27] K. Drewing, M. Fritschi, R. Zopf, M. O. Ernst, and M. Buss, "First evaluation of a novel tactile display exerting shear force via lateral displacement," ACM transactions on Applied Perception, vol. 2, no. 2, pp. 118-131, 2005

[28] V. Levesque, J. Pasquero, and V. Hayward, "Braille display by lateral skin deformation with the stress tactile transducer," in Proceedings of World Haptics 2007, 2007.

[29] Q. Wang and V. Hayward, "In vivo biomechanics of the fingerpad skin under local tangential traction," Journal of Biomechanics, vol. 40, no. 4 , pp. 851-860, 2007.

[30] V. Levesque, J. Pasquero, V. Hayward, and M. Legault, "Display of virtual braille dots by lateral skin deformation: Feasibility study," $A C M$ Transactions on Applied Perception, vol. 2, no. 2.

[31] M. Konyo, K. Akazawa, S. Tadokoro, , and T. Takamori, "Tactile feel display for virtual active touch," in Proceedings of the 2003 IEEE/RSJ International Conference on Intelligent Robots and Systems Las Vegas, Nevada, October, 2003, pp. 3744-3750.

[32] K.-U. Kyung, M. Ahn, D.-S. Kwon, and M. A. Srinivasan, "A compact planar distributed tactile display and effects of frequency on texture judgement," Advanced robotics, vol. 20, no. 5, pp. 563-580, 2006.

[33] S. Tadokoro, S. Yamagami, M. Ozawa, T. Kimura, T. Takamori, and K. Oguro, "Multi-dof device for soft micromanipulation consisting of soft gel actuator elements," in Proc. IEEE ICRA, 1999, pp. 2177-2182.

[34] Y. Ikei and M. Shiratori, "Textureexplorer: A tactile and force display for virtual textures," in Proceeding of the 10th Symp. On haptic Interfaces for Virtual Environment and Teleoperator Systems (HAPTICS'02), March, Orlando, FL, USA, 2002, pp. 327-334.

[35] M. Fritschi, M. O. Ernst, and M. Buss, "Integration of kinesthetic and tactile display- a modular design concept," in Proceedings EuroHaptics 2006, 2006, pp. 607-612.

[36] C. R. Wagner, D. P. Perrin, O. Clatz, H. Delingette, and N. Ayache, "Integrating tactile and force feedback with finite element models," in Proceedings of the 2005 IEEE International Conference on Robotics and Automation, 2005, pp. 3942- 3947.

[37] W. R. Provancher, K. J. Kuchenbecker, G. Niemeyer, and M. R. Cutkosky, "Perception of curvature and object motion via contact location feedback," International Journal of Robotics Research, vol. 24, no. 9 , pp. 691-702, 2005

[38] C. Ramstein, "Combining haptic and braille technologies: Design issues and pilot study," in Proceedings of the second annual ACM conference on Assistive, Vancouver, British Columbia, Canada.

[39] T. Nobels, G. Desmet, J. V. den Keybus, and R. Belmans, "Development of a portable braille display using a fast prototyping platform for power electronics," in Power Electronics, Machines and Drives, 2006. The 3rd IET International Conference on.

[40] K. J. Kuchenbecker, W. R. Provancher, G. Niemeyer, and M. R. Cutkosky, "Haptic display of contact location," in Haptic Interfaces for Virtual Environment and Teleoperator Systems, 2004. HAPTICS '04. Proceedings. 12th International Symposium on.

[41] P. Kammermeier, A. Kron, J. Hoogen, and G. Schmidt, "Display of holistic haptic sensations by combined tactile and kinesthetic feedback," Presence, vol. 13, no. 1, pp. 1-15, 2004.

[42] K. Kim, M. C. Cavusoglu, and W. K. Chung, "Quantitative comparison of bilateral teleoperation systems using $\mathcal{H}_{\infty}$ framework," in IEEE/RSJ International Conference on Intelligent Robots and Systems, Sendai, Japan, 2004, pp. 2229- 2234.

[43] — - "Quantitative comparison of bilateral teleoperation systems using $\mu$-synthesis," IEEE Transactions of Robotics, vol. 23, no. 4, pp. 776789, 2007.

[44] Sensing systems co. [Online]. Available: http://www.sensingsystems.com/

[45] S. Bolanowski Jr, G. Gescheider, R. Verrillo, and C. Checkosky, "Four channels mediate the mechanical aspects of touch," The Journal of the Acoustical Society of America, vol. 84, p. 1680, 1988.

[46] S. Bolanowski, G. Gescheider, and R. Verrillo, "Hairy skin: Psychophysical chnnels and their physiological substrates," Somatosensory and Motor Research, vol. 11, no. 3, pp. 279-290, 1994. 